OPEN

SUBJECT AREAS:

TWO-DIMENSIONAL

MATERIALS

QUANTUM DOTS

Received

17 September 2013

Accepted

30 October 2013

Published

15 November 2013

Correspondence and requests for materials should be addressed to X.S.X. (xsxu@semi.ac.

$\mathrm{cn})$

\section{Enhanced trion emission from colloidal quantum dots with photonic crystals by two-photon excitation}

Xingsheng $X_{u}$

State Key Laboratory of Integrated Optoelectronics, Institute of Semiconductors, Chinese Academy of Sciences, Beijing 100083, China.

For colloidal quantum dots, the ongoing biggest problem is their fluorescence blinking. Until now, there is no generally accepted model for this fluorescence blinking. Here, two-photon excited fluorescence from $\mathrm{CdSe} / \mathrm{ZnS}$ nanocrystals on silicon nitride photonic crystals is studied using a femtosecond laser. From analysis of the spectra and decay processes, most of the relative trion efficiency is larger than $10 \%$, and the largest relative trion efficiency reaches $46.7 \%$. The photonic crystals enhance the trion emission of CdSe/ZnS nanocrystals, where the enhancement is due to the coupling of the trion emission to the leaky mode of the photonic crystal slab. Moreover, the photonic crystals enhance the Auger-assisted trapping efficiency of electrons/holes to surface states, and then enhance the efficiency of the generations of charge separation and DC electric field, which modifies the trion spectrum. Therefore, a model is present for explaining the mechanism of fluorescence blinking including the effect of the environment.

luorescence intermittency is one of the few remaining challenging problems in quantum mechanisms, and it is a significant weakness for applications in quantum information. Photoluminescence (PL) intermittency is mainly interpreted as being caused by charging and discharging of nanocrystals ${ }^{1}$, where charged quantum dots (QDs) are weak emitters due to fast nonradiative Auger recombination of excitons. However, some other models that are supported by experimental results and theoretical analysis indicate that fluorescence intermittency may be triggered by random activation of nonradiative exciton traps at the surface of nanocrystals ${ }^{2}$. Researchers measured the lifetime of negatively charged cores as being of the order of tens of microseconds in nanocrystal films, and it was found that nanocrystal photoionization occurs through Auger processes ${ }^{3}$. Measurement of PL decay has provided directly the lifetime of trion emission. It was found that the lifetime of a trion is indeed longer than that of a bi-exciton and also that its radiative rate is faster than the exciton radiative rate at room temperature. The results imply a 10-15\% quantum yield for trions that may be too bright for the off state seen in blinking ${ }^{4}$. An intermediate state was observed in the blinking of single CdSe/CdS core-shell nanocrystals. This state has a low quantum yield higher than the "off" state and much lower than the "on" state observed in the PL blinking of individual nanocrystals. The observed values of those states are consistent with photoinduced, adsorption/desorption events that take place at the surface of the nanocrystals ${ }^{5}$. The surface state results in the dim state facilitating the transition to the off state and an increase of nonradiative relaxation due to the formation of trion emission.

Controlling spontaneous emission from QDs by PhCs is attractive and useful. The effective control of spontaneous emission by photonic crystals (PhCs) has been extensively studied ${ }^{6-8}$. Spontaneous emission from QDs is strongly modified as the photonic band gap covers the QD emission wavelength range ${ }^{9}$. Interaction between highQ PhC nanocavity and spontaneous emission from QDs has been exploited ${ }^{10,11}$. Modification of spectra was found, as well as extraction of spontaneous emission from $\mathrm{PhCs}^{12,13}$. $\mathrm{PhC}$ patterns can enhance the external quantum efficiency of spontaneous emission ${ }^{13-19}$. Most of the literature cited above is related to linearly excited fluorescence, and there are few reports on two-photon excited fluorescence (TPF) controlled by $\mathrm{PhCs}^{20}$. Here, the significant modification of spectra for TPF is observed and analysed, and a blue shift of the spectrum of TPF from CdSe QDs on SiN PhCs is found. This blue shift is attributed to positive trion ${ }^{3-5}$ emission and the coupling of the emission into the leaky mode of the $\mathrm{PhC} \mathrm{slab}^{20}$. This is the first report of the enhancement of charged exciton (trion) emission by two-dimensional PhCs. Both the spectrum and the decay process of QDs on PhCs directly demonstrate that the relative trion efficiency is higher than $10 \%$, which is too bright for the off state observed in the blinking phenomenon. It has been proved that for a emitter with emission located at the photonic band, the 
radiative rate changes less than $20 \%{ }^{21}$. PhCs provide us with a new tool to explore the photo-physics mechanism for fluorescence blinking of single emitters.

\section{Results}

TPF spectra from QDs on PhCs. Amorphous silicon nitride ( $\mathrm{SiN}$ ) $\mathrm{PhCs}$ and CdSe QDs were prepared as described elsewhere ${ }^{22}$. A fabricated $\mathrm{PhC}$ is shown in Fig. S1. We measured the spectra of TPF from CdSe/ZnS QDs on PhCs with different lattice constants (see methods). The spectra of QDs on PhCs are fitted to a Gaussian function; some fitting curves and corresponding original experimental data are shown in Fig. S2. The coincidence between the experimental data and the Gaussian function differs for the different lattice constants. For lattice constants of $1250 \mathrm{~nm}$ and $700 \mathrm{~nm}$ (representing a significant blue shift), both the right- and left-hand sides of the spectra cannot be fitted well to the Gaussian function. For the lattice constant of $950 \mathrm{~nm}$, the right-hand side of the spectrum fits relatively well to the Gaussian function, but on the left-hand side, there is a weak spectral peak, which is more distinct for the spectrum for the lattice constant of $1250 \mathrm{~nm}$. The coincidence for the fitting of the spectra for lattice constants of $700 \mathrm{~nm}$ or $650 \mathrm{~nm}$ is worse, although their PL intensities are much lower than that for the lattice constant of $1250 \mathrm{~nm}$. The spectra for lattice constants of $1500 \mathrm{~nm}$ or $900 \mathrm{~nm}$ can be fitted fairly well to the Gaussian function, and there is no obvious peak on the short-wavelength side.

The normalized spectra from the QDs on PhCs and those on blank $\mathrm{SiN}$ material are compared in Fig. 1, which shows normalized spectra for PhC lattice constants of $1250 \mathrm{~nm}, 1000 \mathrm{~nm}, 800 \mathrm{~nm}, 750 \mathrm{~nm}$, $700 \mathrm{~nm}$ and $600 \mathrm{~nm}$, and the spectrum from QDs without $\mathrm{PhC}$. The profiles of the spectra are different for PhCs with different lattice constants. Compared with the spectra from QDs on blank SiN without PhCs, the spectra from QDs on PhCs undergo a blue shift, and the shift values vary with the $\mathrm{PhC}$ lattice constant. The average centre wavelength of the spectrum from QDs on blank without $\mathrm{PhC}$ is $586.38 \mathrm{~nm}$. For a lattice constant of $1250 \mathrm{~nm}$, the central wavelength of the spectrum from this $\mathrm{PhC}$ is $582.50 \mathrm{~nm}$ by fitting with a Gaussian function, and the blue shift is $3.9 \mathrm{~nm}$ compared with the blank. For lattice constants of $1000 \mathrm{~nm}$ and $800 \mathrm{~nm}$, the central peak wavelengths are $581.12 \mathrm{~nm}$ and $575.79 \mathrm{~nm}$, and the blue shifts are $5.2 \mathrm{~nm}$ and $10.6 \mathrm{~nm}$, respectively. For a lattice constant of $700 \mathrm{~nm}$, the centre wavelength is $570.70 \mathrm{~nm}$ and the blue shift is $15.6 \mathrm{~nm}$, which is the largest shift among these PhCs. To confirm the shift, the spectra of QDs in Fig. 1 were fitted to BiGaussian function (sum of two terms of a Gaussian function) for comparison.

The coincidence of the spectra with the single Gaussian function is not high, which may indicate that PL from QDs does not decay via a single pathway. Therefore, we fitted the spectra from QDs on PhCs to a BiGaussian function (sum of two terms of a Gaussian function), as shown in Fig. 2. It can be seen that all the spectra from QDs on PhCs can be fitted well to BiGaussian functions, except those for $\mathrm{PhCs}$ with lattice constants of $900 \mathrm{~nm}$ and $750 \mathrm{~nm}$, which can be fitted well to a single Gaussian function. After fitting, the spectra from QDs on PhCs can be divided into two parts, one is a long component at a long wavelength, and the other is a short component at a short wavelength. The long component is denoted as X emission, while the short component is denoted as $\mathrm{X}^{*}$ emission.

The parameters for the spectra from QDs on PhCs fitted to the BiGaussian function depend on the lattice constant, and they are shown in Fig. 3. From Fig. 3a, the centre wavelengths of both the $\mathrm{X}$ emission and the $\mathrm{X}^{*}$ emission of QDs on PhCs are shorter than those of the spectra without PhCs. As analysed above, by fitting to a single Gaussian function, the spectra of QDs on PhCs shift to the blue compared with those on the blank. The central wavelengths of both $\mathrm{X}$ and $\mathrm{X}^{*}$ vary with $\mathrm{PhC}$ lattice constant. The variation range of the centre wavelength (deviation between the maximum and minimum in the wavelength range $577.93-585.69 \mathrm{~nm}$ ) of $\mathrm{X}$ is $7.7 \mathrm{~nm}$, while

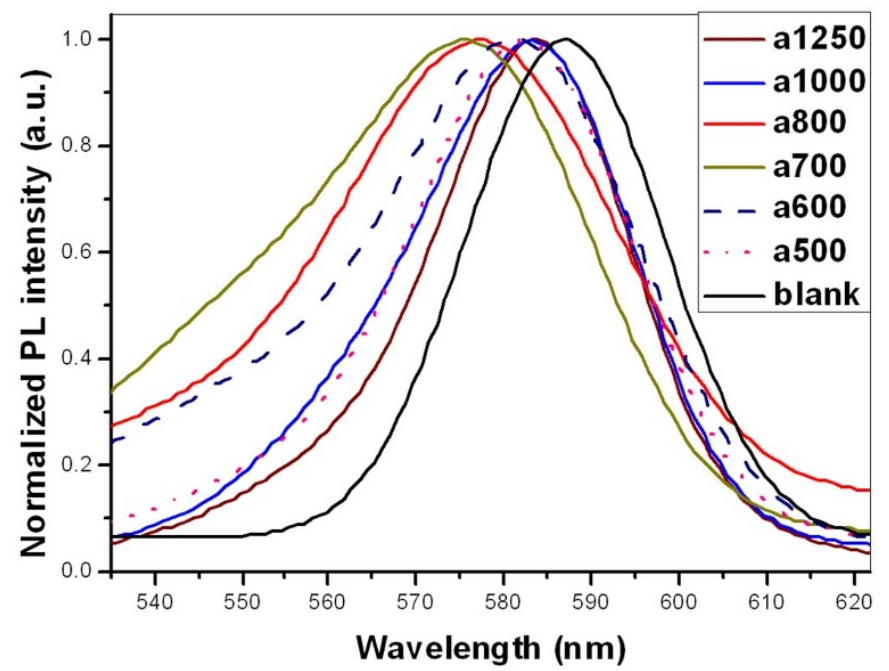

Figure 1 The TPF spectra from QDs on PhCs and blank. Normalized TPF spectra from QDs on PhCs with lattice constants of $1250 \mathrm{~nm}$, $1000 \mathrm{~nm}, 800 \mathrm{~nm}, 750 \mathrm{~nm}, 700 \mathrm{~nm}$ and $600 \mathrm{~nm}$, compared with that from QDs on blank SiN; the spectrum from the blank is averaged from four spectra of QDs in different positions of blank SiN without PhCs. The values represent the lattice constants; "blank" means the SiN membrane without $\mathrm{PhC}$.

that (in the range $446.01-578.89 \mathrm{~nm}$ ) of $\mathrm{X}^{*}$ reaches $32.9 \mathrm{~nm}$. Compared to $\mathrm{X}^{*}$, the variation of the centre wavelength of $\mathrm{X}$ is relatively stable. The largest blue shift of $40.38 \mathrm{~nm}$ (deviation between the centre wavelength of the spectra of QDs on PhCs and that on blank) for $\mathrm{X}^{*}$ is for the lattice constant of $800 \mathrm{~nm}$, where the enhancement factor is only around 4. For lattice constants of $1000 \mathrm{~nm}$ and $1250 \mathrm{~nm}$, the enhancement factor is larger than 40 and the blue shift of $X^{*}$ is only around $10.9 \mathrm{~nm}$. The spectral width for $X$ and $X^{*}$ is shown in Fig. $3 b$, where the spectral width of $X^{*}$ is much broader than that of $\mathrm{X}$. The spectral width of $\mathrm{X}^{*}$ is around $19 \mathrm{~nm}$, while that of $X$ is around $11 \mathrm{~nm}$. The spectral widths of $X$ and $\mathrm{X}^{*}$ are similar for lattice constants of $500 \mathrm{~nm}$ and $550 \mathrm{~nm}$. Moreover, the overall variation tendency is that the spectral width slightly decreases with increasing lattice constant. The ratio of the $\mathrm{X}^{*}$ and $\mathrm{X}$ components is shown in Fig. $3 \mathrm{c}$. The ratio of the $\mathrm{X}$ component is larger than 0.5 , while that of $\mathrm{X}^{*}$ is less than 0.5 but larger than 0.1 . Therefore, the relative efficiencies of $\mathrm{X}^{*}$ are all larger than $10 \%$, and the largest reaches $46.7 \%$. The large ratios of the $\mathrm{X}$ component are at lattice constants of $500 \mathrm{~nm}, 550 \mathrm{~nm}$ and $800 \mathrm{~nm}$, with values of 0.88 , 0.87 and 0.85 , respectively. In contrast, the corresponding ratios of the $\mathrm{X}^{*}$ component at the same lattice constants are small. To some extent, the shorter the centre wavelength of $\mathrm{X}^{*}$, the smaller the ratio of the component of $\mathrm{X}^{*}$. From the decay process that can be fitted to a tri-exponential function, the ratio of multi-exciton to exciton emission is about 0.25 (from the result of QDs on SiN membranes). However, in the experiment measuring TPF spectra, the detector is a CCD whose response time is longer than $1 \mathrm{~ns}$; thus the detected TPF spectra do not include multi-exciton emission. From the characterization of TPF spectra, the $\mathrm{X}$ component is exciton emission, while the $\mathrm{X}^{*}$ component, thus the large blue shift of the TPF spectra of QDs on PhCs, is attributed to positive trion (charged exciton) emission $^{3}$. Time-resolved PL experiments will demonstrate further that the short $\mathrm{X}^{*}$ component from the BiGaussian fitting is the emission from a trion. The trion state leads to a reduction in PL in nanocrystals on these PhCs. The surface state results in a dim state facilitating the transition to the off state and an increase of nonradiative relaxation due to the formation of trion emission ${ }^{5}$. In PhCs with different lattice constants, the local density of states (LDOS) is different, and thus the electric fields corresponding to excitation light 

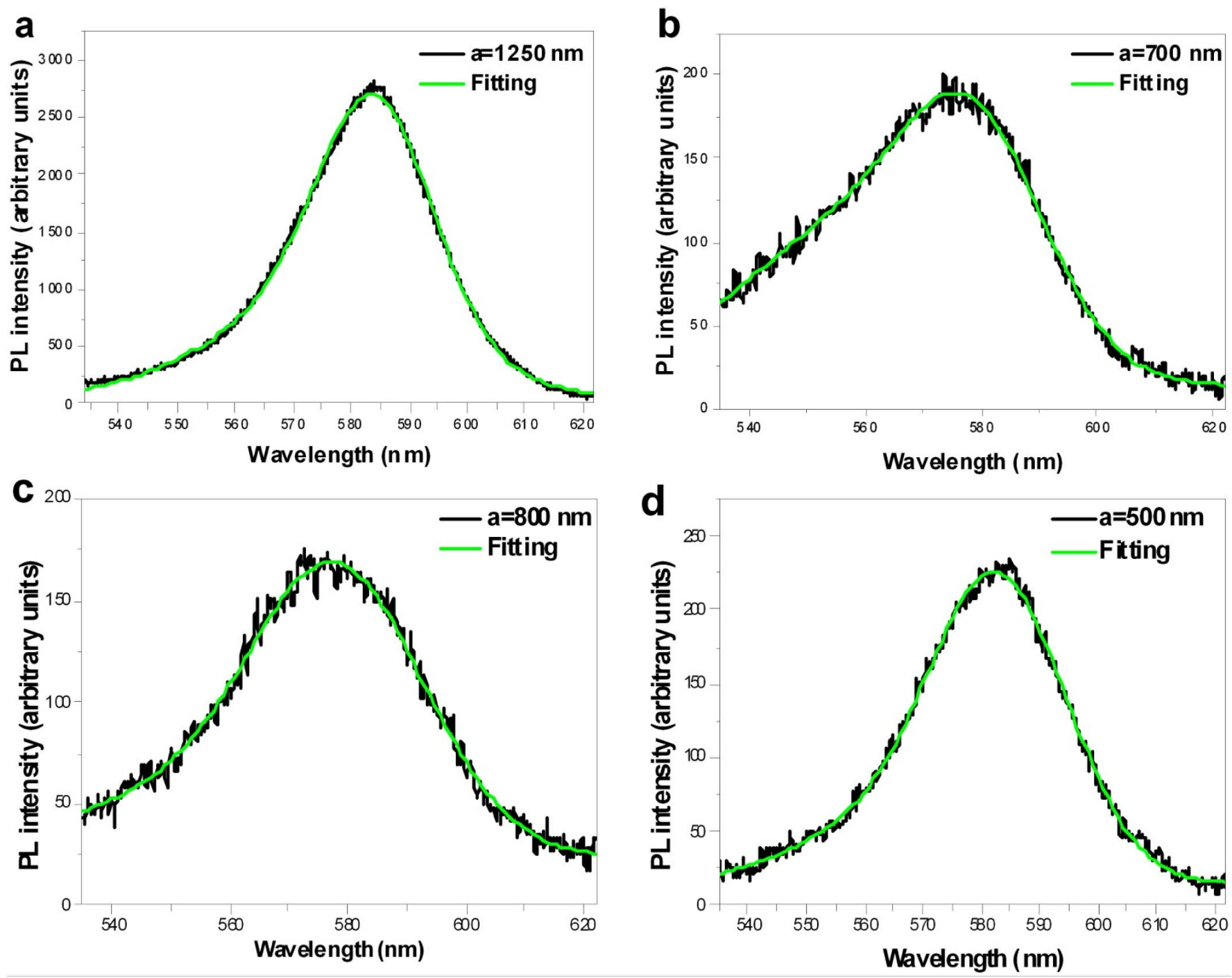

Figure $2 \mid$ Spectra from QDs on PhCs fitted by BiGaussian functions. The PhC lattice constants are (a) $1250 \mathrm{~nm}$, (b) $700 \mathrm{~nm}$, (c) $800 \mathrm{~nm}$ and (d) $500 \mathrm{~nm}$. The spectra are measured at the same excitation intensity. The black lines are the TPF spectra measured from QDs on PhCs, and the green lines are the fitting lines of BiGaussian functions.

and emission light are different. Therefore, the LDOS in PhCs will change the ionization states, and then the emission wavelength of charged exciton (trion) varies with lattice constant, as shown in Fig. 3a. Figure $3 c$, $d$ shows that the relative efficiency of trion $X^{*}$ is larger than $10 \%$ and the ratios of the component of trion $\mathrm{X}^{*}$ to that of exciton $\mathrm{X}$ on these $\mathrm{PhCs}$ are larger than 0.1 , the largest ratio reaching 0.87 for a lattice constant of $1000 \mathrm{~nm}$. Due to the similar emission level, the trion and the exciton may be explored as a two-photon entangled source. This ratio is much larger than that predicated by the charging model for blinking, where the trion represents the off state, while the exciton represents the on state in the charging model.

Mechanism to the asymmetry of TPF spectra. From Figs. 2 and 3, the intensity of TPF depends on the lattice constants of PhCs, and TPF from QDs on PhCs is highly enhanced compared to that without PhCs. The enhancement mechanism is that excitation light and emission light are resonant with the leaky mode of the PhC $\operatorname{slab}^{14,20}$. We correlate the TPF enhancements with the presence of photonic modes near the $\Gamma$ point for the peak emission wavelength of $585 \mathrm{~nm}$, and note that the pump wavelength of $800 \mathrm{~nm}$ is resonant with the leaky mode of the PhC slab. The field enhancement is explained by considering the photonic band structure of the PhCs shown in Fig. 4. Two points should be mentioned. The first is that the spectral width of the femtosecond laser is not so narrow. As measured, the full width at half maximum reaches about $10 \mathrm{~nm}$. This means that as we use a femtosecond laser with a centre wavelength of $800 \mathrm{~nm}$, the actual pump wavelength ranges from about $795 \mathrm{~nm}$ to $805 \mathrm{~nm}$. The second point concerns the irradiation and collection angle of pump light and emission light, respectively. We used an objective lens with a magnification of $40 \times$. The irradiation and collection angle is about $-2.3^{\circ}$ to $2.3^{\circ}$, the $k$ vector ranges from 0 to $0.04\left(\sin \left(2.3^{\circ}\right)\right)$, which is shown by the vertical dotted line in the band structure in Fig. 4 . For a lattice constant of $1250 \mathrm{~nm}$, the pump wavelength of $800 \mathrm{~nm}$ corresponds to the normalized frequency of $1.5625 \mathrm{a} / \lambda$, and is indicated as point $\mathrm{A}$ at $\mathrm{a}$ leaky mode of the PhC slab in Fig. 4. For a lattice constant of $1000 \mathrm{~nm}$, the pumping wavelength of $800 \mathrm{~nm}$ corresponds to the normalized frequency of $1.25 a / \lambda$, and is indicated by point $B$ at a leaky mode of the $\mathrm{PhC}$ slab. Therefore, the electric fields of the pump light in PhCs with lattice constants of $1250 \mathrm{~nm}$ and $1000 \mathrm{~nm}$ are significantly enhanced and the ratio of trion is large. However, there is also a leaky mode at a normalized frequency of $1.0625 a / \lambda(a=$ $850 \mathrm{~nm}$ at pump wavelength of $800 \mathrm{~nm}$ ), while the ratio of trion for the $\mathrm{PhC}$ with lattice constant of $850 \mathrm{~nm}$ is smaller. The reason is that, for lattice constants of $1250 \mathrm{~nm}$ and $1000 \mathrm{~nm}$, there are also leaky 

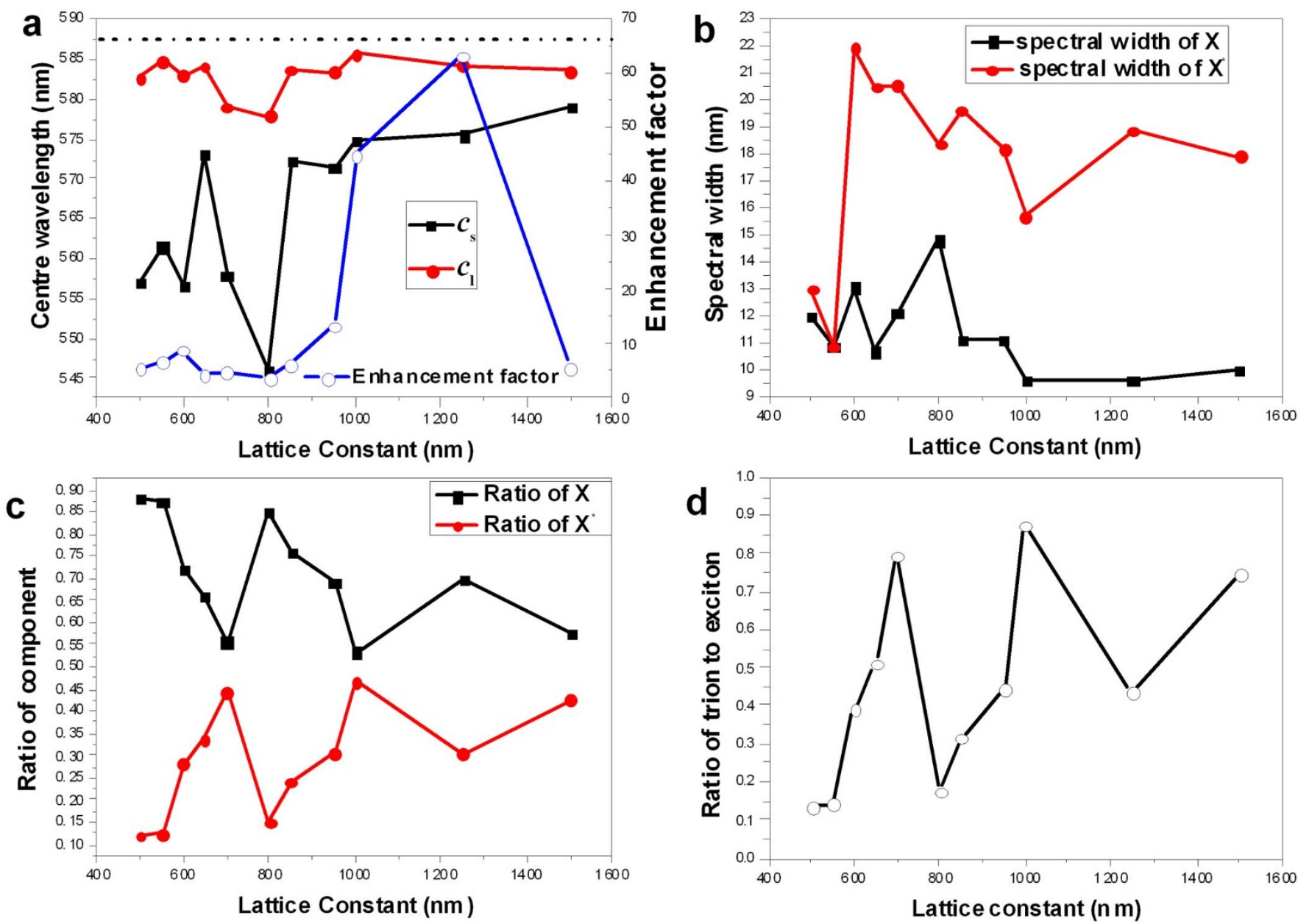

Figure 3 The parameters from the spectra fitted by BiGaussian functions for different PhC lattice constants. (a) Centre wavelength of spectra fitted to a BiGaussian function of QDs on PhCs with different lattice constants, where $c_{\mathrm{l}}$ and $c_{\mathrm{s}}$ are the centre wavelengths of the long and short components, respectively. Integrated enhancement factor for different lattice constants is shown. Dotted line represents the centre wavelength of spectrum on SiN film without PhCs. (b) Spectral width. (c) Ratio of X and X* components. These ratios represent the relative emission efficiency of X and X*. (d) Ratio of X* to $\mathrm{X}$.

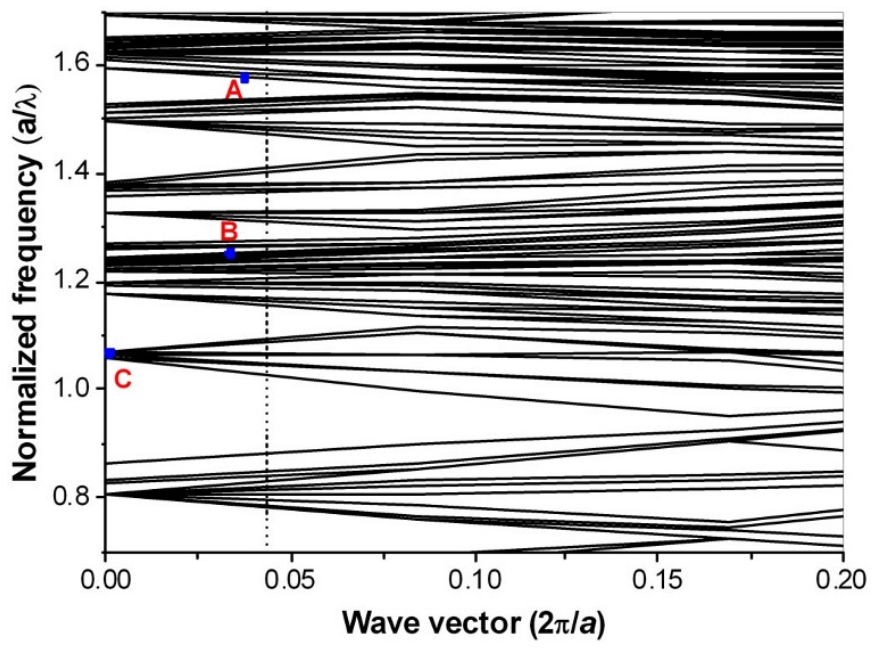

Figure $4 \mid$ Band structure of the PhC. The blue spots A (1.5625a/ $\lambda)$, B $(1.25 a / \lambda)$ and $\mathrm{C}(1.0625 \mathrm{a} / \lambda)$ correspond to the leaky modes of the PhC slab with respective lattice constants of $1250 \mathrm{~nm}, 1000 \mathrm{~nm}$ and $850 \mathrm{~nm}$ at an excitation wavelength of $800 \mathrm{~nm}$. modes in the band structure overlapping or nearly overlapping with the main emission band around $585 \mathrm{~nm}$, while for the lattice constant of $850 \mathrm{~nm}$, there is no corresponding mode.

To study the enhancement of the spectra of QDs on PhCs, the ratio of the PL intensity of QDs on PhCs to that on blank without PhCs is calculated (See Fig. S3). For different lattice constants ranging from $1500 \mathrm{~nm}$ to $500 \mathrm{~nm}$, the peaks of the enhancement factor are located at approximately the same wavelength, which is centred around 555$558 \mathrm{~nm}$ with broad spectral width. The enhancement peaks at 555$558 \mathrm{~nm}$ in Fig. S3 is attributed to the trion emission. It is difficult to find leaky modes in the band structure that meet the same peak with different lattice constants.

To demonstrate the above analysis, time-resolved PL was measured over a 50 ns time range from the same QDs on SiN membrane by using a streak scope (see methods). The spectra with an excitation power of $300 \mu \mathrm{W}$ at delay of $0 \mathrm{~ns}$ integrated in a 950 ps gate (curve $F$ ), a delay of 1 ns at a gate of $1 \mathrm{~ns}$ (curve $M$ ), and a delay of $15 \mathrm{~ns}$ at a gate of $4.5 \mathrm{~ns}$ (curve $S$ ) were recorded and shown, respectively, as black, red and blue lines in Fig. 5a. Curve $F$ contains multi-exciton emission and single exciton, and curve $M$ contains trion emission and single exciton ${ }^{23}$. By fitting the spectra to a Gaussian function, the centre wavelength of curve $S$ (exciton emission) is $594.5 \mathrm{~nm}$ with a spectral width of $15.52 \mathrm{~nm}$ and the centre wavelength of curve $M$ is $589.30 \mathrm{~nm}$ with a spectral width of $17.3 \mathrm{~nm}$. From Fig. 5a, the spectrum of curve $M$, thus the trion spectrum is shifted to the blue 

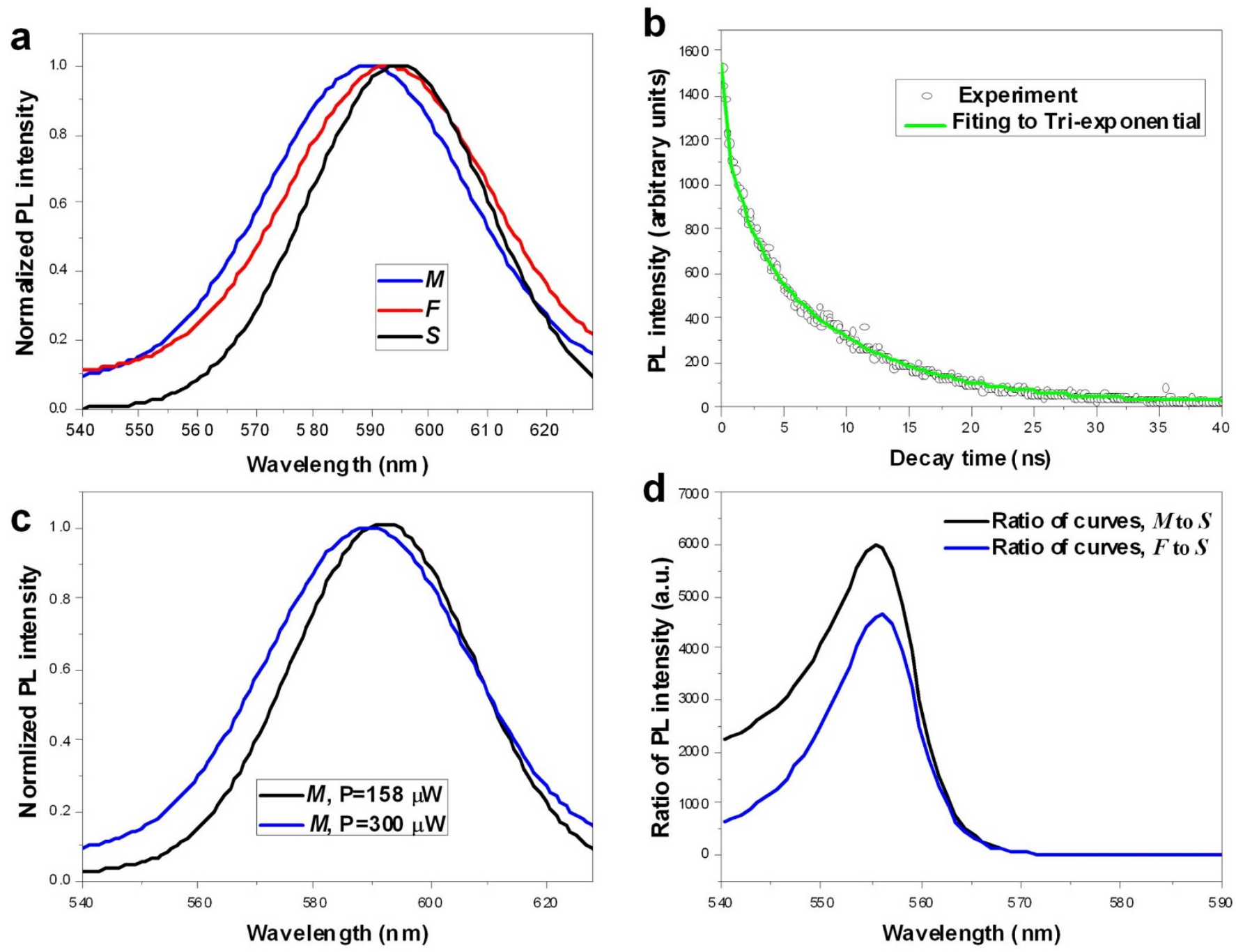

Figure 5 |TPF spectra and the decay process of QDs on SiN material measured using a streak camera. (a) Time-resolved PL under $300 \mu \mathrm{W}$ excitation over 50 ns. Curve $F$ at delay 0 ns, integrated in 950 ps, curve $M$ at delay 1 ns gate 1 ns and curve $S$ at delay 15 ns gate 4.5 ns, respectively. (b) Decay process of QDs on SiN membrane, corresponding to the PL in a. (c) Time-resolved spectra at delay 1 ns gate 1 ns (curve Min a) at different excitation intensities under $800 \mathrm{~nm}$ femtosecond laser excitation. (d) Ratios of curves $F$ and $M$ to $S$.

compared with that of exciton. The decay process of PL from QDs on $\mathrm{SiN}$ membrane is shown in Fig. 5b. The green line is a fitted triexponential curve, where obtained lifetimes of $8.91 \mathrm{~ns}, 1.77 \mathrm{~ns}$ and $0.28 \mathrm{~ns}$ represent lifetimes of exciton, trion and multi-exciton, respectively. From the fitted amplitude in Fig. 5b, the ratio of trion to exciton reaches $43 \%$, and the relative trion efficiency is $12.8 \%$. This result is in accordance with that obtained from the PL spectra in Fig. $3 \mathrm{c}$. In a previous study ${ }^{4}$, by measuring the PL decay process, a $10-15 \%$ quantum yield for trion emission was obtained. Here, by studying both PL decay and PL spectra, we obtain a trion efficiency larger than $10 \%$ from CdSe/ZnS QDs on $\mathrm{SiN}$ membranes or on $\mathrm{SiN}$ $\mathrm{PhC}$ slabs, and the largest trion efficiency for QDs on PhCs reaches $46.7 \%$. Moreover, from Fig. $5 c$, as the excitation intensity increases, the M-spectrum, thus the spectrum of the trion emission shifts to the blue. The ratios of the PL intensity of curve $F$ and that of $M$ to that of $S$ are shown in Fig. 5d. The spectral intensities of curve $M$ and curve $F$ in the short-wavelength region are enhanced significantly compared with those of the exciton, and both ratio peaks are at around $555 \mathrm{~nm}$, which is in accordance with the ratio of PL spectra from QDs in Fig. S3. Fig. S4 shows the time-resolved TPF spectra of QDs on PhCs measured using a streak camera, where the spectrum of curve $M$, thus the trion spectrum is shifted to the blue compared with that of exciton, and the PL intensity of curve $M$ (thus the trion spectrum) in the short wavelength region is much stronger than that of exciton spectrum.

Some previous reports revealed that photo-oxidation by oxygen and water molecules results in a large blue shift for CdSe/ZnS nanocrystals. However, the blue shift is not due to photo-oxidation in our study because the measurement conditions for PhCs and the blank without PhCs are the same. If this blue shift was a result of photooxidation, the size of the QD core would reduce, and at the same time, the PL intensity would decrease ${ }^{24}$. In contrast, we observed that the two-photon PL intensity increased with an increase in the blue shift of the spectra.

\section{Discussion}

In conclusion, under two-photon excitation, the QDs absorb twophoton light and emit visible light around $585 \mathrm{~nm}$. Comparison of the spectra of QDs on PhCs with those on blank material indicates that the TPF from QDs on PhCs is significantly enhanced. The electric field for the excitation wavelength is enhanced because the LDOS in the PhCs is enhanced compared with that in blank material. The enhanced electric fields of the excitation light and emission light result in both enhancements of the intensity of TPF and blue shift of the TPF spectrum. In PhCs, excitation intensity is enhanced, and under the relatively strong field of excitation light in PhCs, which 
will introduce the DC Stark effect ${ }^{25}$, the charged exciton emission is generated. Moreover, due to the two-photon excitation energy being above the band gap of CdSe, the optical Stark effect ${ }^{26}$ may also take effect. Therefore, the blue shift of TPF spectra of QDs on PhCs is mainly attributed to charged exciton (trion) emission. It has been proved that for emitters in $\mathrm{PhC}$ membranes, the lifetime of emission in the photonic band region is similar to or only decreases to approximately $80 \%$ of that of the blank material without $\mathrm{PhCs}^{21}$. $\mathrm{PhC}$ membranes will not decrease much the emission lifetime of QDs directly, but the PhCs enhance the excitation intensity, the trion and multiexciton emission efficiency. Therefore, the enhancement of trion emission is mainly due to the change in QD microscopic structure and decay path of PL. The time-resolved experiments demonstrate further that the blue shift of spectra of QDs on PhCs mainly arises from charged exciton (trion) emission. On some PhCs, the ratio of trion to exciton reaches 0.87 , which means that the charging model is not suitable here, and the enhancement is due to the trion emission coupling to the leaky mode of the PhC slab. Therefore, the model of the mechanism for blinking on PhCs should combine the charging model with the enhancement effect of PhCs. This means that PhCs enhance the trion PL intensity and also modify the spectrum. This process is believed to be caused by the enhancement of the electric field introduced by the charge separation between the charge in the nanocrystal core and that of the surface states. PhCs enhance the Auger-assisted trapping efficiency of electrons/holes to surface states, and then enhance the efficiency of charge separation and generation of DC electric field that modifies the trion spectrum. Therefore, PhCs enhance the DC Stark effect created by charge separation, and the different DC Stark effect in PhCs results in different trion emission intensity and emission wavelength.

TPF from CdSe/ZnS nanocrystals on PhCs undergoes a blue shift when compared with that without PhCs. The maximum blue shift of the TPF spectra is $40 \mathrm{~nm}$. The largest ratio of the trion to exciton components reaches $87 \%$, and the high efficiency of the trion may make it possible to explore excitons and trions as a two-photon entangled source. The blue shift and enhancement of the TPF PL intensity are due to coupling of pump light and TPF emission to the leaky modes of the PhC slab. The asymmetry of the PL spectra from $\mathrm{PhCs}$ with different lattice constant is due to combined effect of enhancement from PhCs and trion emission from colloidal QDs. The strong electric field of the pump light and TPF in the PhCs results in an enhancement of the Auger-assisted trapping efficiency of electrons/holes to surface states, and the efficiency of charge separation and generation of a DC electric field, and then positive trion emission takes place. This phenomenon indicates that trion emission and blinking of colloidal nanocrystals depend on the environment; combining the charging model and $\mathrm{PhC}$ enhancement effect can explain the blinking phenomenon of QDs on PhCs. Therefore, we deduced that a model for QDs blinking should include Auger process and Auger-assisted surface recombination.

\section{Methods}

In the measurement of TPF from colloidal QDs, a femtosecond laser with a pulse width of around $80 \mathrm{fs}$, repetition frequency of $80 \mathrm{MHz}$ and wavelength of $800 \mathrm{~nm}$ was focused onto the sample with a $40 \times$ objective lens at room temperature. The TPF was collected by the same objective lens in the vertical direction. The average excitation power before the objective lens was $56 \mathrm{~mW}$. The emission light was collected into a spectrometer equipped with a silicon CCD (Hamamatsu).

Time-resolved PL was measured over a 50 ns time range from the same QDs on $\mathrm{SiN}$ membrane by using a streak scope (Hamamatsu). A femtosecond laser with a pulse width of $120 f s$, repetition frequency of $1 \mathrm{kHz}$ and wavelength of $800 \mathrm{~nm}$ is focused by a $20 \times$ objective lens. The sample was colloidal CdSe QDs on a SiN film. The average excitation power before the objective lens was $300 \mu \mathrm{W}$.

Two-dimensional photonic crystals on SiN membrane were fabricated by electron beam lithography and reactive ion etching ${ }^{22}$. The thickness of SiN film was $160 \mathrm{~nm} \mathrm{SiN}$ film, which was deposited on a silicon substrate using plasma-enhanced chemical vapor deposition. The refractive index of the SiN material was 1.99. The lattice constants ranged from $400 \mathrm{~nm}$ to $1500 \mathrm{~nm}$. After dry etching, the silicon under SiN membrane was removed by HNA (HF:Nitric:Acetic) mixture to form a free-standing structure. The colloidal quantum dots were casted onto the surface of SiN photonic crystals.
Fitting of the spectra was done using Origin 8.0 software. To investigate the physical mechanism behind the asymmetry of the PL spectrum, the measured spectrum was fitted to a BiGaussian function

$$
y=y_{0}+A_{l} \exp \left(-\frac{\left(x-c_{l}\right)^{2}}{2 \omega_{l}^{2}}\right)+A_{s} \exp \left(-\frac{\left(x-c_{s}\right)^{2}}{2 \omega_{s}^{2}}\right),
$$

where $A_{1}$ and $A_{s}$ are the amplitudes of the two Gaussian components, $\omega_{1}$ and $\omega_{\mathrm{s}}$ are the spectral widths, $c_{1}$ and $c_{\mathrm{s}}$ are the centre wavelengths, and $y_{0}$ is the relative amplitude.

1. Efros, A. L. \& Rosen, M. Random telegraph signal in the photoluminescence intensity of a single quantum dot. Phys. Rev. Lett. 78, 1110-1113 (1997).

2. Galland, G. et al. Two types of luminescence blinking revealed by spectroelectrochemistry of single quantum dots. Nature 479, 203-208 (2011)

3. Saba, M. et al. Light-induced charged and trap states in colloidal nanocrystals detected by variable pulse rate photoluminescence spectroscopy. ACS Nano 7, 229 (2013).

4. Jha, P. P. \& Guyot-Sionnest, P. Trion decay in colloidal quantum dots. ACS Nano 3, 1011-1015 (2009).

5. Gómez, D. E., van Embden, J., Mulvaney, P., Fernée, M. J. \& Rubinsztein-Dunlop, $\mathrm{H}$. Exciton-trion transitions in single CdSe-CdS core-shell nanocrystals. ACS Nano 3, 2281-2287 (2009).

6. Lodahl, P. et al. Controlling the dynamics of spontaneous emission from quantum dots by photonic crystals. Nature 430, 654 (2004).

7. Fujita, M., Takahashi, S., Tanaka, Y., Asano, T. \& Noda, S. Simultaneous inhibition and redistribution of spontaneous light emission in photonic crystals. Science 308, 1296 (2004).

8. Wijinhoven, J. E. G. J. \& Vos, W. L. Preparation of photonic crystals made of air spheres in titania. Science 281, 802 (1998)

9. Yamaguchi, M., Fujita, M., Asano, T., Nakanishi, J. \& Noda, S. Investigation of spontaneous emission from quantum dots embedded in two-dimensional photonic-crystal slab. Electron. Lett. 41, 1402 (2005).

10. Hennessy, K. et al. Quantum nature of a strongly coupled single quantum dotcavity system. Nature 445, 896 (2007).

11. Badolato, A. et al. Deterministic coupling of single quantum dots to single nanocavity modes. Science 308, 1158 (2005).

12. Ganesh, N. et al. Enhanced fluorescence emission from quantum dots on a photonic crystal surface. Nat. Nanotechnol. 2, 515 (2007).

13. Ren, F.-F. et al. Strong vertical light output from thin silicon rich oxide/ $/ \mathrm{SiO}_{2}$ multilayers via in-plane modulation of photonic crystal patterns. Appl. Phys. Lett. 93, 091901 (2007).

14. Fan, S., Villeneuve, P. R., Joannopoulos, J. D. \& Schubert, E. F. High extraction efficiency of spontaneous emission from slabs of photonic crystals. Phys. Rev. Lett. 78, 3294 (1997).

15. Boroditsky, M. et al. Light extraction from optically pumped light-emitting diode by thin-slab photonic crystals. Appl. Phys. Lett. 75, 1036 (1999).

16. Erchak, A. A. et al. Enhanced coupling to vertical radiation using a twodimensional photonic crystal in a semiconductor light-emitting diode. Appl. Phys. Lett. 78, 563 (2001).

17. Ryu, H. Y., Lee, Y. H., Sellin, R. L. \& Bimberg, D. Over 30-fold enhancement of light extraction from free-standing photonic crystal slabs with InGaAs quantum dots at low temperature. Appl. Phys. Lett. 79, 3573 (2001).

18. Kim, J. Y. et al. Enhanced light extraction from GaN-based green light-emitting diode with photonic crystal. Appl. Phys. Lett. 91, 181109 (2007).

19. Kim, S. H. et al. Fabrication of photonic crystal structures on light emitting diodes by nanoimprint lithograph. Nanotechnology 18, 055306 (2007).

20. Inoue, S. I. \& Yokoyama, S. Enhancement of two-photon excited fluorescence in two-dimensional nonlinear optical polymer photonic crystal waveguides. Appl. Phys. Lett. 93, 111110 (2008).

21. Fujita, M., Takahashi, S., Tanaka, Y., Asano, T. \& Noda, S. Stimulated inhibition and redistribution of spontaneous light emission in photonic crystals. Science 308, 1296 (2005).

22. Xu, X. S., Yamada, T., Ueda, R. \& Otomo, A. Two-photon excited fluorescence from CdSe quantum dots on SiN photonic crystals. Appl. Phys. Lett. 95, 221113 (2009).

23. Marceddu, M. et al. Charged excitons, Auger recombination and optical gain in CdSe/CdS nanocrystals. Nanotechnology 23, 015301 (2012).

24. Wang, X., Zhang, J., Nazzal, A. \& Xiao, M. Photo-oxidation-enhanced coupling in densely packed CdSe quantum-dot films. Appl. Phys. Lett. 83, 162 (2003).

25. Klimov, V. I. \& McBranch, D. W. Auger-process-induced charge separation in semiconductor nanocrystals. Phys. Rev. B 55, 13173 (1997).

26. Schaller, R. D., Agranovich, V. M. \& Klimov, V. I. High-efficiency carrier multiplication through direct photogeneration of multi-excitons via virtual single-exciton states. Nature Phys. 1, 189-194 (2005).

\section{Acknowledgments}

We acknowledge financial support from the National Basic Research Program under Grant No 2013CB632105, the National Natural Science Foundation of China under Grant Nos 91121019,61275045 and 61021003 . 


\section{Additional information}

Supplementary information accompanies this paper at http://www.nature.com/ scientificreports

Competing financial interests: The author declares no competing financial interests.
How to cite this article: Xu, X.S. Enhanced trion emission from colloidal quantum dots with photonic crystals by two-photon excitation. Sci. Rep. 3, 3228; DOI:10.1038/srep03228 (2013)

(c) (1) $\Theta$ This work is licensed under a Creative Commons Attribution-

cc. visit http://creativecommons.org/licenses/by-nc-nd/3.0 